\title{
CAMBIOS EN LA FRECUENTACIÓN DE PATOLOGÍAS EN CONSULTA EXTERNA. ANÁLISIS DE LA EVOLUCIÓN EN 6 AÑOS
}

\author{
J. CABALlERO ALCÁNTARA, M. PADILlA LEÓN, C. MARCHAL ESCALONA, \\ J. GARCÍA PENIT
}

Servicio de Urología. Hospital Costa del Sol. Marbella (Málaga).

Actas Urol Esp. 28 (2): 95-100, 2004

\section{RESUMEN}

CAMBIOS EN LA FRECUENTACIÓN DE PATOLOGÍAS EN CONSULTA EXTERNA. ANÁLISIS DE LA EVOLUCIÓN EN 6 AÑOS

INTRODUCCIÓN: En 1996 realizamos un análisis de la tasa de frecuentación de patologias atendidas en la consulta externa. A lo largo de estos años hemos realizado diversas estrategias de actuación sobre algunas patologías o entidades como la HBP y la contracepción masculina.

OBJETIVOS: Conocer el resultado de dichas medidas pasados 6 años, en el año 2002.

MATERIAL Y MÉTODO: Se analiza el porcentaje de las distintas patologías vistas en consulta externa de la Unidad de Urología en el año 2002, y se comparan los resultados con los obtenidos antes de haber realizado el rediseño de la actuación sobre los casos mencionados.

RESULTADOS: El rediseño en el manejo de la contracepción masculina y de la HBP ha permitido disponer de un $15 \%$ y un $1,2 \%$ más de primeras visitas en consulta externa respectivamente, mientras que se ha incrementado un $25 \%$ la consulta por sospecha de cáncer prostático. Se aprecian importantes incrementos de consultas por patologías como la hematuria $(56,4 \%)$, crisis renoureteral $(51,9 \%)$, síndrome miccional $(61,1 \%)$ e impotencia $(17,8 \%)$.

CONCLUSIONES: El rediseño de la actuación sobre patologías es una herramienta eficaz en la gestión de una Unidad de Urología. Su análisis permite valorar los beneficios conseguidos y detectar nuevos problemas que surgen.

PALABRAS CLAVE: Gestión. Frecuentación. Rediseño.

\section{ABSTRACT \\ FREQUENCY CHANGES OF ATTENDED PATHOLOGIES IN EXTERN OFFICE. EVOLUTION ANALYSIS IN SIX YEARS}

INTRODUCTION: In 1996 we made a rate frequency analysis of attended pathologies in extern office. Along these years, we have made differents actuations about some pathologies like HBP and vasectomy.

OBJETIVES: To know the results of these measures after 6 years, in 2002 .

MATERIAL AND METHODS: Different pathologies frequency is analyzed in extern office of Urology in 2002 and the results are compared with those we got before making the remake of the action about the mentioned cases.

RESULTS: The remake in the treatment of the vasectomy and HBP let us to have a $15 \%$ and $1.2 \%$ more of first visits in extern office respectively. While the first visits because of suspicion of prostatic cancer has been increased in $25 \%$. Visits because of pathologies like hematuria, renal colic, cystitis and impotency have been increased importantly $(56.4 \%, 51.9 \%, 61.1 \%$ and $17.8 \%)$.

CONCLUSIONS: The remake of actions on pathologies is a efficacious tool in the management of an Urology Area. Its analysis let us to value the benefits we have got and to detect new problems that appear.

KEY WORDS: Management. Frequentation. Remake. 
A nte una demanda de servicios cada vez mayor y la consecuente sobrecarga asistencial, se han de desarrollar fórmulas de gestión adecuadas a unos recursos que siempre son limitados.

Para responder a las prioridades y responsabilidades de los pacientes, clínicos y gestores, es de gran utilidad la gestión clínica o managed care, que es un proceso de toma de decisiones en la práctica clínica para conseguir el máximo beneficio para el paciente mejorando la calidad del servicio. Con ella se trata de hacer mejor las cosas, en tiempo y con los recursos apropiados.

La gestión clínica permite potenciar actividades asistenciales que mejoren resultados en niveles de salud, eficiencia, uso de tecnologías y utilización de recursos humanos. Engloba un conjunto de estrategias orientadas a aumentar la eficiencia y la calidad mediante la reducción selectiva de la utilización inapropiada, y tiene un instrumento que es la revisión y la evaluación ${ }^{1,2}$.

Existen diversas técnicas de gestión clínica como son el gatekeeping como cuidados compartidos, el case management o gestión de casos, la gestión de procesos, la reingeniería de procesos, etc., orientados a la consecución de una mejora asistencial continua ${ }^{3-6}$.

Como estableció Juran, en el ciclo de la mejora continua de la calidad, tras tener una visión estratégica del cambio y establecer sus objetivos, se diseña la innovación organizativa y se redisenan los procesos, estableciendo posteriormente una comparativa o benchmarking interno $\mathrm{y} / \mathrm{o}$ externo para evaluar el resultado del cambio ${ }^{7-9}$

La tasa de frecuentación hospitalaria es un índice definido por la relación entre el número de ingresos realizados en un hospital durante un año y la población a la que atiende éste ${ }^{6}$.

Trasladando la definición anterior a la actividad de un Servicio de Urología, y más concretamente a la frecuentación de patologías en la consulta externa como casos nuevos, podemos tener una tasa de frecuentación de consulta externa ${ }^{6}$.

Esta tasa va a depender de diversos factores. Sobre algunos es difícil que podamos actuar, como son los factores demográficos, epidemiológicos, socioeconómicos: pirámide de edad poblacional, nivel cultural, etc. Pero sobre otros si tenemos más capacidad de actuación y gestión, como son el grado asistencial desarrollado por la asistencia primaria, el grado de coordinación interniveles en materia de diagnóstico y tratamiento, etc. ${ }^{6}$

El análisis de esta tasa de frecuentación y su evolución en el tiempo nos permite conocer la evolución que va teniendo en el tiempo la frecuentación de las distintas patologías y el impacto que sobre ello conllevan las distintas actuaciones de gestión acometidas a lo largo de los años.

En realidad será un instrumento más del sistema de información global del que debemos disponer para el eficaz funcionamiento de nuestra unidad.

\section{MATERIAL Y MÉTODO}

En el presente trabajo se realiza un análisis de la frecuentación de las distintas patologías que son atendidas en la consulta externa de la Unidad de Urología del Hospital Costa del Sol en el año 2002.

Este Hospital cubre la asistencia de 240.000 habitantes y el trabajo se basa en la coordinación interniveles, fundamentalmente con Asistencia Primaria.

La recogida de datos se realiza sobre el libro de registro de consulta externa donde se escribe el diagnóstico inicial de primera visita que el urólogo dictamina según historia clínica, exploración y pruebas complementarias de que dispone el paciente en ese momento.

Los distintos diagnósticos iniciales más frecuentes y comunes recogidos en el libro y analizados en el presente trabajo son los siguientes:

1. Próstata: HPB, carcinoma, prostatitis aguda, prostatitis crónica, hemospermia y otros.

2. Riñón: litiasis renoureteral, pielonefritis, trauma, neoformación, quistes, hidronefrosis, tuberculosis, estenosis de la unión pieloureteral y otros.

3. Vejiga: cistitis, incontinencia, neoformación, enuresis, hematuria-microhematuria (considerando que su origen más frecuente es la vejiga, aunque puede tener otras procedencias), litiasis, reflujo, vejiga neurógena y otros.

4. Teste-epidídimo-cordón: criptorquidia, trauma, infertilidad, quiste, vasectomía, orquiepididimitis, varicocele, hidrocele, neoformación, prótesis y otros. 
5. Pene: fimosis-frenillo corto, impotencia, enfermedad de Peyronie, neoformación, condiloma acuminado, eyaculación precoz y otros.

6. Uretra: hipospadias, trauma, estenosis, estenosis de meato, carúncula y otros ${ }^{10}$.

Una vez calculados los porcentajes de frecuentación de las distintas patologías ocurridos en el año 2002, se realiza una comparativa con los recogidos en un trabajo previo correspondientes al año 1996.

Por último, se realiza un análisis de los cambios ocurridos en la frecuentación de las distintas patologías, tanto de ascenso como de descenso, sus posibles causas y el impacto que haya podido tener el tipo de gestión de nuestra Unidad y las estrategias tomadas durante este periodo de tiempo.

\section{OBJ ETIVOS}

1. Conocer la frecuentación en consulta externa en el año 2002 según el tipo de patología.

2. Realizar una comparación de la frecuentación existente en el año 2002 frente a la que se produjo en el año 1996.

3. Analizar los cambios de incremento y decremento ocurridos en las distintas patologías y sus posibles causas.

4. Analizar la influencia que han podido tener el tipo de gestión desarrollada por la Unidad y las estrategias establecidas.

\section{RESULTADOS}

Los resultados obtenidos en el año 1996 en cuanto a porcentajes de frecuentación por patologías quedan reflejados en la Figura 1, y destacan por su elevada frecuencia y por orden la HPB (16\%), la contracepción (15\%), la litiasis renoureteral $(10,2 \%)$ y fimosis-frenillo (10\%), seguidos a distancia por un grupo de patologías de frecuencia media, la hematuria $(3,9 \%)$, el síndrome cistítico $(3,6 \%)$, el carcinoma de próstata-PSA elevado $(3,3 \%)$, la prostatitis $(3,1 \%)$, la incontinencia $(3 \%)$ y la impotencia $(2,8 \%)$.

En la Figura 2 se reflejan así mismo los correspondientes al año 2002. Destacan por orden de frecuencia: la crisis renoureteral (15,5\%), HPB $(14,8 \%)$, fimosis-frenillo $(8,5 \%)$, hematuria $(6,1 \%)$, síndrome cistítico $(5,8 \%)$, carcinoma de próstataPSA elevado $(4,1 \%)$, impotencia $(3,3 \%)$, prostatitis $(3 \%)$, dolor lumbar $(2,6 \%)$ y la incontinencia $(2,1 \%)$.

\section{DISCUSIÓN}

En este trabajo realizamos el análisis de un aspecto importante de nuestro quehacer diario como es conocer la frecuentación de la patología que atendemos habitualmente, desde la fuente por donde entra dicha patología, que es la consulta externa.

Cuestiones que nos podemos preguntar a diario se pueden responder con este tipo de análisis: ¿Qué patología vemos con más frecuencia?, ¿Qué patología está aumentando su incidencia?, ¿Qué patología está disminuyéndola?, ¿Por qué estos cambios?, ¿Qué podemos hacer para controlar una patología emergente con gran aumento de su frecuentación?, ¿Qué resultados obtenemos de algunas actuaciones estratégicas de gestión?.

Habitualmente realizamos este ejercicio de análisis en distintas facetas de nuestro trabajo, lo que ocurre es que muchas veces no somos conscientes de que lo hacemos, de que estamos aplicando la trilogía de Juran en la búsqueda de la calidad de nuestro trabajo: planificación, control y mejora.

Analizando los resultados obtenidos, apreciamos patologías que descienden su tasa de frecuentación y otras que la incrementan.

Entre las que descienden destacan la contracepción masculina (un 94,7\%), la incontinencia (un 30\%) y la HPB (un 7,5\%) en términos relativos, mientras que en términos absolutos lo serían la primera y la tercera al ser más incidentes.

$\mathrm{Y}$ entre las que incrementan su frecuentación destacan la impotencia (un 17,8\%), la elevación de PSA-cáncer de próstata (un 24,2\%), la litiasis renoureteral (un 51,9\%), la hematuria (un $56,4 \%$ ), el síndrome cistítico (un $61,1 \%$ ) y el dolor lumbar (un 73,3\%) en términos relativos, mientras que en términos absolutos lo sería la litiasis renoureteral por ser más incidente.

Dentro de las medidas estratégicas de funcionamiento desarrolladas por nuestra unidad a lo largo de estos años, destacan la coordinación con la Asistencia Primaria para el diagnóstico y tratamiento de la HPB, y la coordinación con las unidades de Planificación Familiar de Asistencia Primaria para seguir el protocolo de contracepción quirúrgica masculina.

En la primera se establecieron unos parámetros diagnósticos para realizar tratamiento de la HPB y derivación a asistencia especializada cuan- 


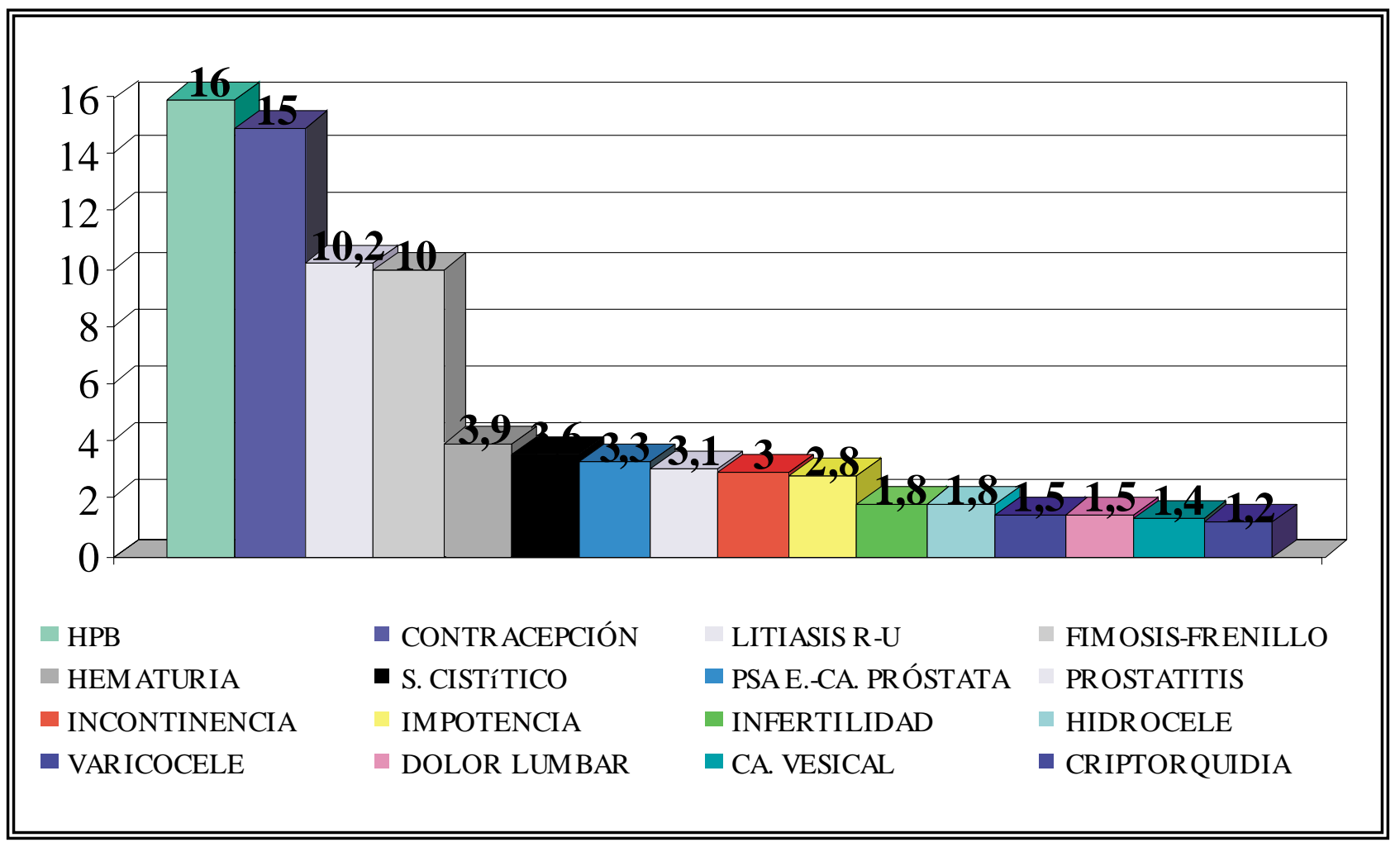

FIGURA 1. Tasa de frecuentación. 1996.

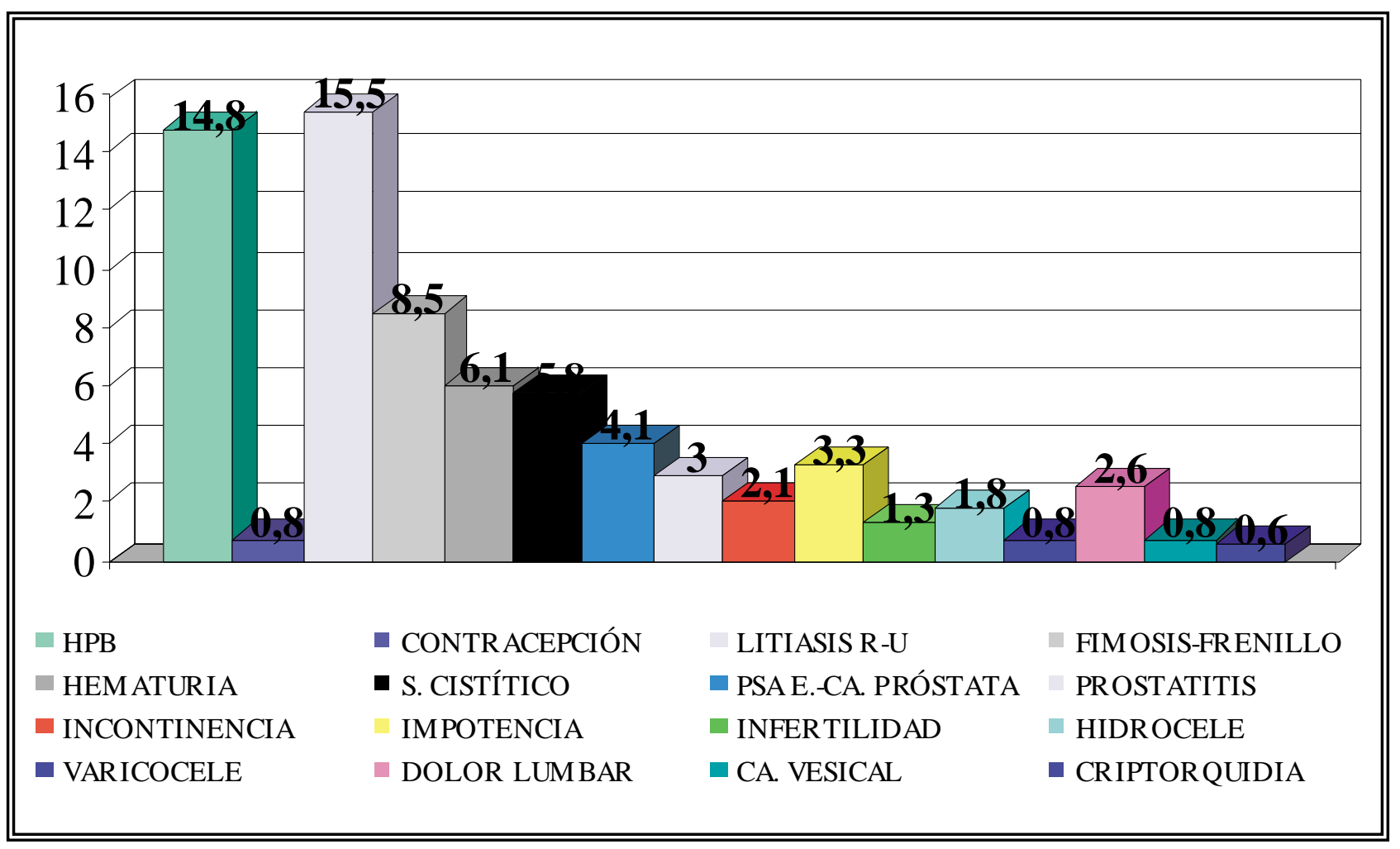

FIGURA 2. Tasa de frecuentación. 2002. 
do se cumplieran determinados criterios. En la segunda se estableció que fueran los centros de Planificación Familiar los que informaran al paciente sobre las posibilidades contraceptivas, tipo de intervención, administración del consentimiento informado y preparación a realizar previa en caso de realización de intervención, de manera que el paciente acude al hospital por primera vez el día en que va a ser intervenido, ahorrándosele así una visita a consultas externas, salvo que desee realizar alguna consulta especial o presente en su historia algún contingente que así lo recomiende.

Comparando los datos obtenidos de los años analizados, observamos que con la primera medida reducimos la frecuentación por HPB en un $7,5 \%$, lo cual nos indica que la coordinación en este aspecto con Asistencia Primaria está siendo efectiva, a la vez que nos permite disponer de más citas de consulta externa libres para control y reducción de su lista de espera. Y además se incrementa la frecuentación por PSA elevado-cáncer de próstata en un $24,2 \%$, lo cual ratifica la eficacia de la coordinación llevada a cabo y la mejora de la calidad del control y diagnóstico de esta patología.

Mientras que con la segunda medida obtenemos una reducción de su frecuentación en un $94,7 \%$, lo cual va a suponer la disposición de gran cantidad de citas libres en consulta externa y reducción de su lista de espera, al ser esta una patología de alta frecuentación en términos absolutos. Y entre las dos medidas, la reducción de uso de consultas para primeras visitas se reduce en un $15,4 \%$ en términos absolutos, lo cual supone casi 2 meses del año.

En cuanto a las patologías que incrementan su frecuentación destaca la impotencia en un $17,8 \%$, debido a que es una patología emergente cada vez de mayor consulta y petición de tratamiento. La hematuria-microhematuria con un incremento del 56,4\%, probablemente debido a que las facilita el tratamiento con anticoagulantes o antiagregantes que se está extendiendo en su uso por diversas causas vasculares periféricas, centrales o neurovasculares. El síndrome cistítico, con un incremento del 61,1\%, y el dolor lumbar y la crisis renoureteral con un $73,3 \%$ y $51,9 \%$ respectivamente, son patologías que destacan por el incremento de frecuentación que han tenido, y que a nuestro parecer es debido a que al no existir especial coordinación entre Asistencia Primaria y Urología en esas patologías, se produzcan derivaciones en mayor cantidad debido probablemente a la presión asistencial (Fig. 3).

En resumen podemos concluir en primer lugar que debemos mantener nuestra actividad coordinada con Asistencia Primaria en las patologías ya establecidas, y en segundo lugar que debemos hacerlo con otras en las que aún no se ha desarrollado y el incremento de frecuentación ha sido notable.

Con este estudio comprobamos como las actuaciones que habitualmente realizamos de rediseño o mejora, control y análisis, para después volver a repetir el ciclo, son útiles (Fig. 4).

\begin{tabular}{|c|c|}
\hline DECREMENTO & INCREMENTO \\
\hline - HPB & - Impotencia \\
\hline \multirow[t]{5}{*}{ - Contracepción } & - PSA elevado-Ca. próstata \\
\hline & - Litiasis R-U \\
\hline & - Hematuria \\
\hline & - Dolor lumbar \\
\hline & - S. Cistítico \\
\hline
\end{tabular}

FIGURA 3. Cambio en frecuentación.

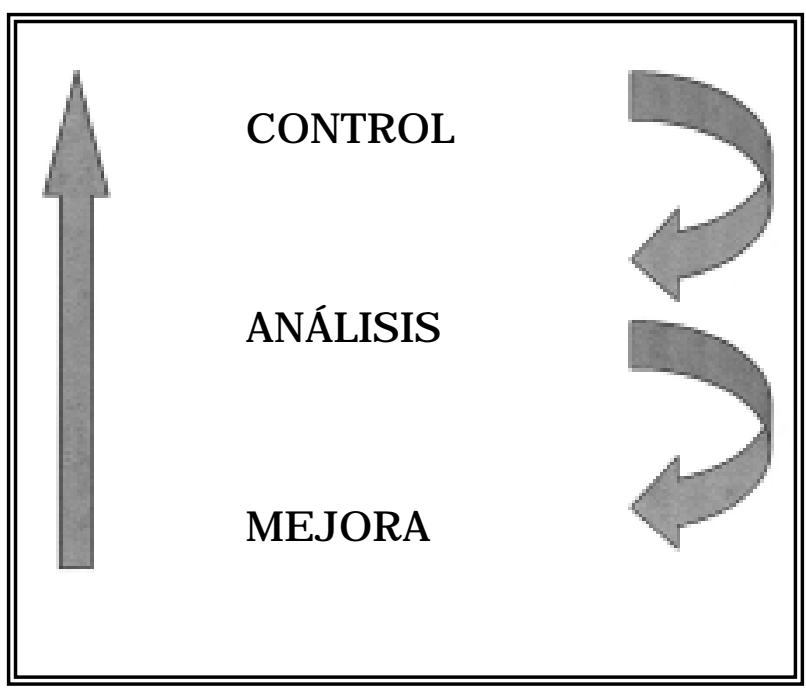

FIGURA 4 
Por un lado nos confirman si los rediseños de procesos que levamos a cabo han sido eficaces, y por otro nos alertan de nuevos problemas que surgen, que nos harán revisar pautas de actuación para así, de forma continua buscar nuestro mejor funcionamiento y resultados.

\section{REFERENCIAS}

1. DEL LLANO J.: Gestión sanitaria y clínica basada en la evidencia. Medicina basada en la evidencia y la práctica clínica en Urología. Ed. Jarpio 2002: 9-22.

2. IBERN P, MENEU R.: Managed care y gestión de la utilización. Gestión sanitaria. Innovaciones y desafíos. Ed. Masson 1998: 219-239.

3. CABALLERO J, PADILLA M, MARCHAL C.: Coordinación protocolizada con la asistencia primaria: nuevo sistema de trabajo en Urología. Su validación. Actas Urol Esp 1998; 22: 111-115.

4. CABALLERO J, MARCHAL C, PADILLA M, RODRIGO I.: Aumento de la tasa cruda de la incidencia del cáncer de próstata en nuestro medio. Actas Urol Esp 2001; 25: 624-627.

5. PADILLA M, MARCHAL C, CABALLERO J, PADILLA F.: Cuidados compartidos en HPB. Primera experiencia nacional. Actas Urol Esp 1998; 22: 478-484.
6. CABALlERO J, PADILlA M, MARCHAL C.: Tasa de frecuentación de consulta externa. Base organizativa y de gestión de un Servicio de Urología. Actas Urol Esp 1998: 22 (3): 215-222.

7. OTEO L, HERNÁNDEZ J.: Rediseño de/en organizaciones sanitarias: de la teoría a la práctica. Gestión sanitaria. Innovaciones y desafíos. $E d$. Masson 1998: 433-458.

8. MARQUET R.: Gestión de la calidad. Innovaciones y desafíos. Ed. Masson 1998: 241-275.

9. SOCHALSKI J, AIKEN I, FAGIN C.: Hospital reestructuring in the United States, Canada and Western Europe. Medical Care 1997; 35: 13-25.

10. ROMERO F.: Signos, síntomas y sindromes en Urología. Urología. Vesalio. Ed. ENE 1992: 67.

Dr. J. Caballero Alcántara

Servicio de Urología

Hospital Costa del sol

Ctra. Nacional 340

29600 Marbella (Málaga)

(Trabajo recibido el 17 octubre de 2003) 